\title{
Tautomeric and aggregational dynamics of curcumin-supersaturated Pluronic ${ }^{\circledR}$ nanocarriers
}

Gustavo Braga $a^{a^{*}}$ Katieli da Silva Souza Campanholi ${ }^{a}$, Sabrina Barbosa de Souza Ferreira ${ }^{b}$, Italo Rodrigo Calori ${ }^{a}$, Jean Halison de Oliveira ${ }^{c}$, Douglas Vanzin ${ }^{a}$, Marcos Luciano Bruschi ${ }^{b}$, Rodrigo Meneghetti Pontes ${ }^{d}$, Paulo Henrique Março ${ }^{e}$, André Luiz Tessaro ${ }^{a, f}$, Noboru Hioka ${ }^{a}$, and Wilker Caetano ${ }^{a}$.

aDepartment of Chemistry, Research Nucleus in Photodynamic Systems, State University of Maringá, Av. Colombo 5790, Maringá, Paraná 87020-900, Brazil.

bepartment of Pharmacy, Laboratory of Research and Development of Drug Delivery Systems, State University of Maringá, Av. Colombo 5790, Maringá, Paraná 87020-900, Brazil. 'Departmen of Chemistry, Federal Technological University of Paraná, Av. João Miguel Caram, 3131, Londrina, Paraná, 86036-370, Brazil.

dDepartment of Chemistry, State University of Maringá, Av. Colombo 5790, Maringá, Paraná 87020-900, Brazil.

ePost-Graduation Program of Food Technology, Federal Technological University of Paraná, R. Rosalina Maria Ferreira, 1233 - Vila Carola, Campo Mourão, Paraná, 87301-899 Brazil.

\section{Gustavo Braga}

*Corresponding author email: gbraga2@uem.br; braga.gustavo@outlook.com

Department of Chemistry, Research Nucleus in Photodynamic Systems (NUPESF), State University of Maringá, Av. Colombo 5790, Maringá, Paraná 87020-900, Brazil.

\section{Supporting Information}


A<smiles>COc1cc(/C=C/C(=O)CC(=O)/C=C/c2ccc(O)c(OC)c2)ccc1O</smiles><smiles>COc1cc(/C=C/C2=CC(=O)OC(C(F)(F)F)O2)ccc1O</smiles>

B
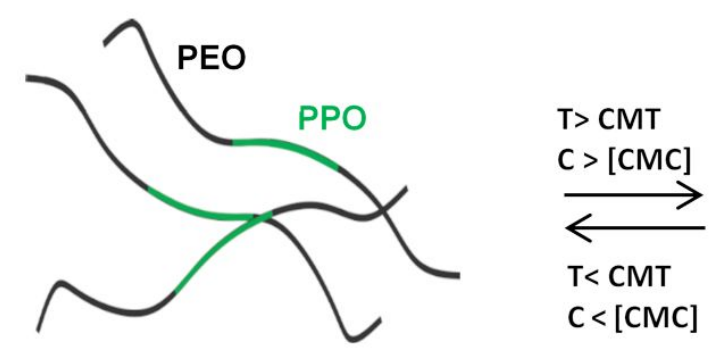

C<smiles>[Y]C(C)(O)COC(C)(C)C(C)(C)CC(C)OC([Y])(C)COC(C)C</smiles>
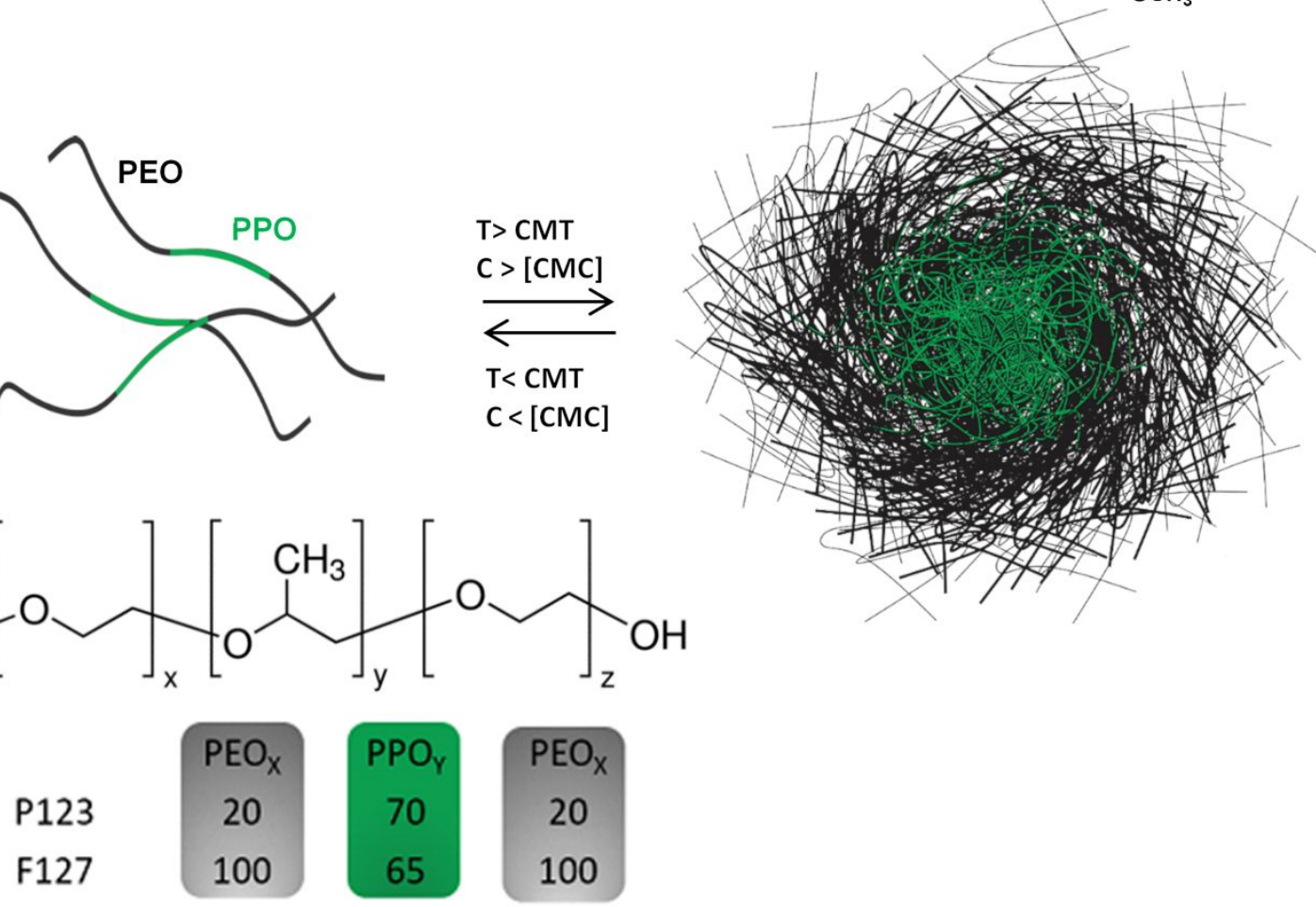

Figure S1. A. Cur Keto-Enolic equilibrium. B. Representation of the formation of copolymeric micelles in aqueous solution, $\mathrm{T}=$ Temperature, $c m t=$ critical micellar temperature, $[\mathrm{cmc}]=$ critical micellar concentration. C. Structural formula of Pluronics ${ }^{\circledR}$, composed of poly(ethylene oxide)-block-poly(propylene oxide)-block-poly(ethylene oxide) (PEOx-PPOy-PEOx) groups; $\mathrm{x}$ are the numbers of poly(ethylene oxide) groups and $\mathrm{y}$ are the poly(propylene oxide) groups. $\mathrm{P} 123\left(\mathrm{PEO}_{20}-\mathrm{PPO}_{70}-\mathrm{PEO}_{20}\right)$ groups, and F127 $\left(\mathrm{PEO}_{100} \mathrm{PPO}_{65}-\mathrm{PEO}_{100}\right)$ groups. 
Table S1. Total electronic energy for the optimized structures of copolymeric fragments and copolymeric segments with Cur tautomers calculated at the HF-3c and B3LYP/6$31+\mathrm{G}(\mathrm{d}, \mathrm{p}) / / \mathrm{HF}-3 \mathrm{c}$ levels of theory in vacuum medium

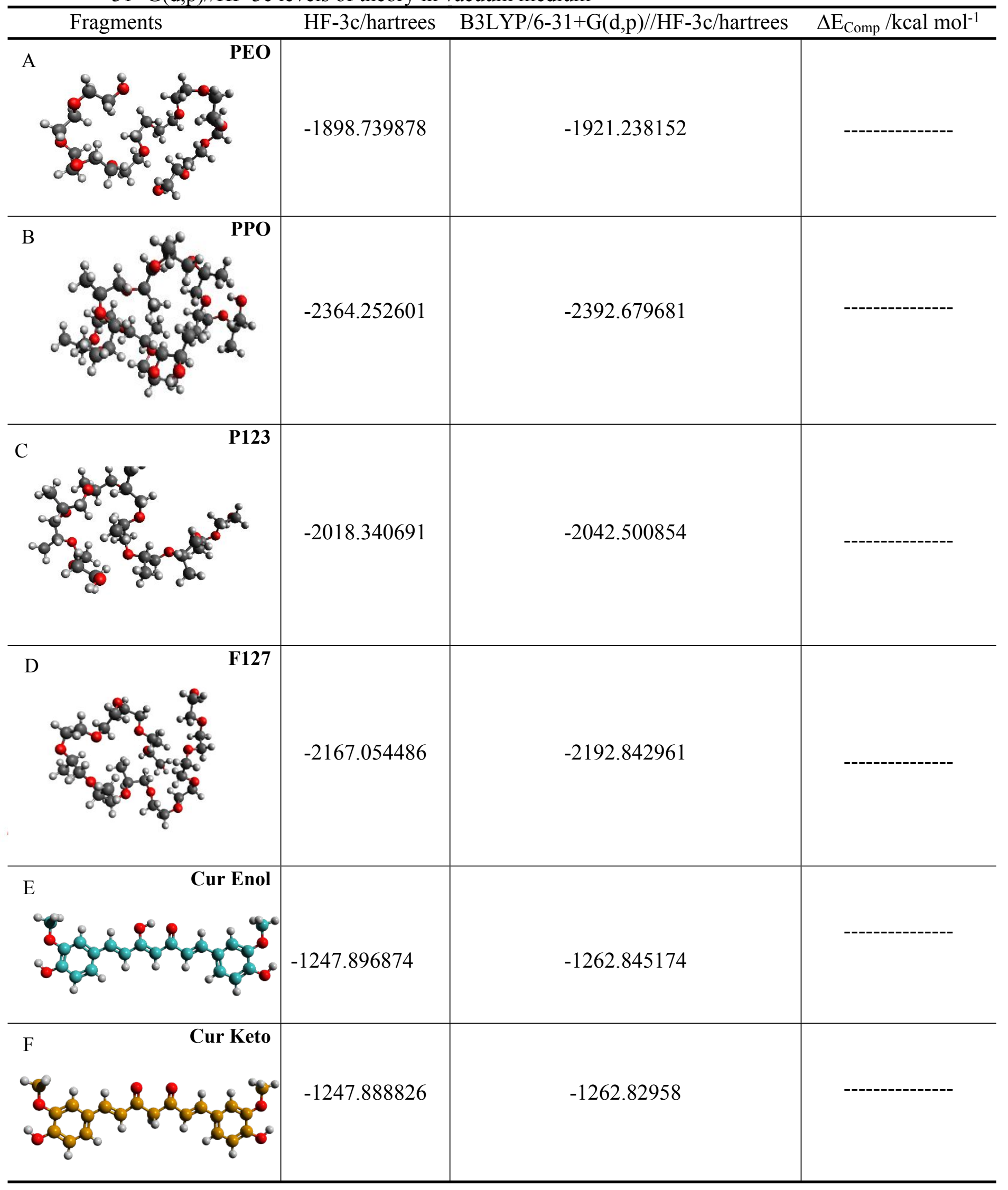


Table S2. Total electronic energy for the optimized structures of copolymeric fragments and copolymeric segments with Cur tautomers calculated at the HF-3c and B3LYP/6$31+\mathrm{G}(\mathrm{d}, \mathrm{p}) / / \mathrm{HF}-3 \mathrm{c}$ levels of theory in vacuum medium

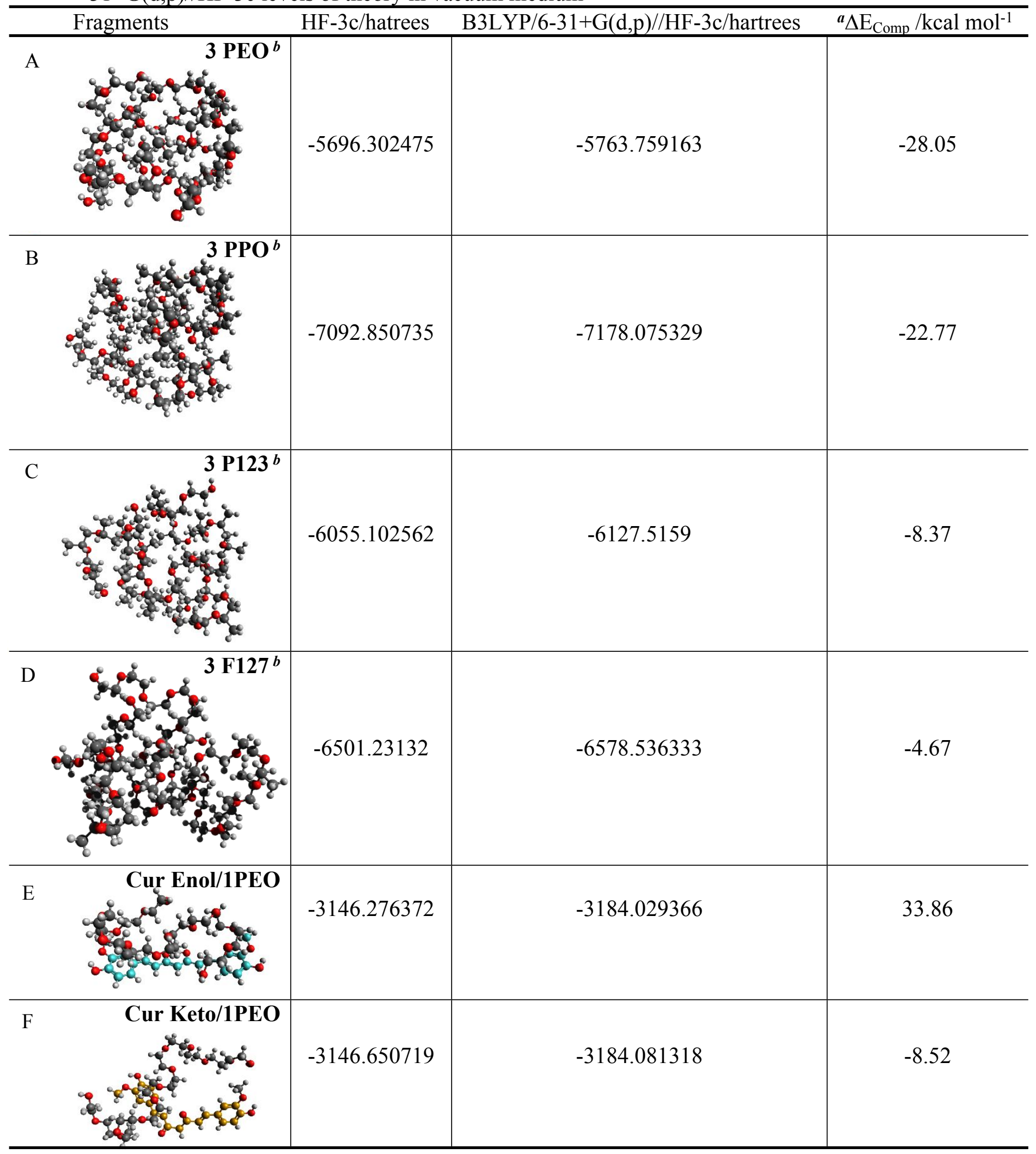

1 hartree $=627.5095 \mathrm{kcal} / \mathrm{mol}$.

${ }^{a}$ For the calculations of $\Delta E_{\text {Comp }} / k_{c a l ~ m o l}{ }^{-1}$ were used the values from $B 3 L Y P / 6-31+G(d, p) / / H F-3 c / h a r t r e e s$.

${ }^{b}$ These energy values are from the total amount of copolymers fragments optimized together. 
Table S3. Total electronic energy for the optimized structures of copolymeric fragments and copolymeric segments with Cur tautomers calculated at the HF-3c and B3LYP/6$31+\mathrm{G}(\mathrm{d}, \mathrm{p}) / / \mathrm{HF}-3 \mathrm{c}$ levels of theory in vacuum medium

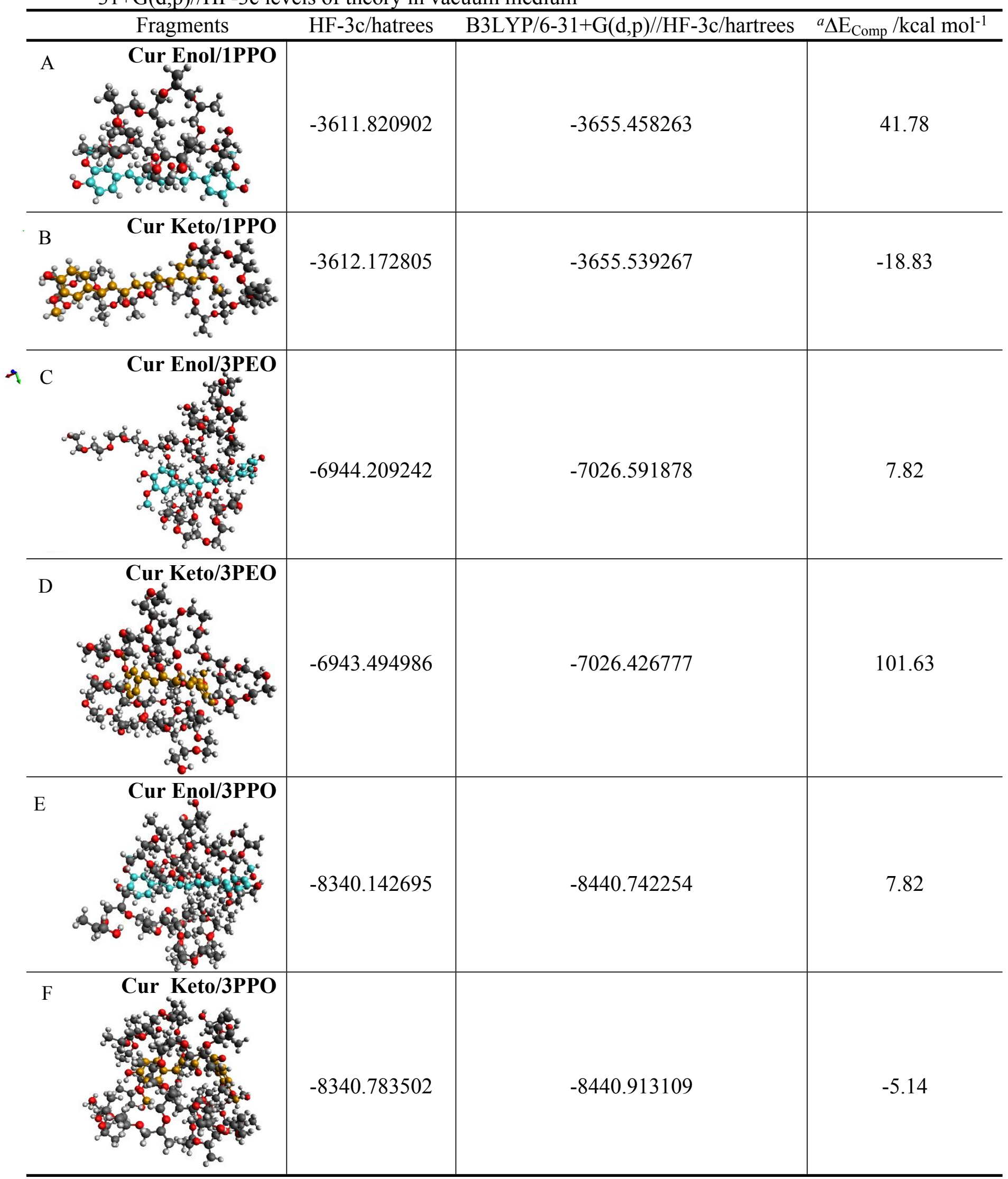

1 hartree $=627.5095 \mathrm{kcal} / \mathrm{mol}$.

${ }^{a}$ For the calculations of $\Delta E_{\text {Comp }} / k c a l$ mol $^{-1}$ were used the values from $B 3 L Y P / 6-31+G(d, p) / / H F-3 c / h a r t r e e s$. 
Table S4. Total electronic energy for the optimized structures of copolymeric fragments and copolymeric segments with Cur tautomers calculated at the HF-3c and B3LYP/6$31+\mathrm{G}(\mathrm{d}, \mathrm{p}) / / \mathrm{HF}-3 \mathrm{c}$ levels of theory in vacuum medium

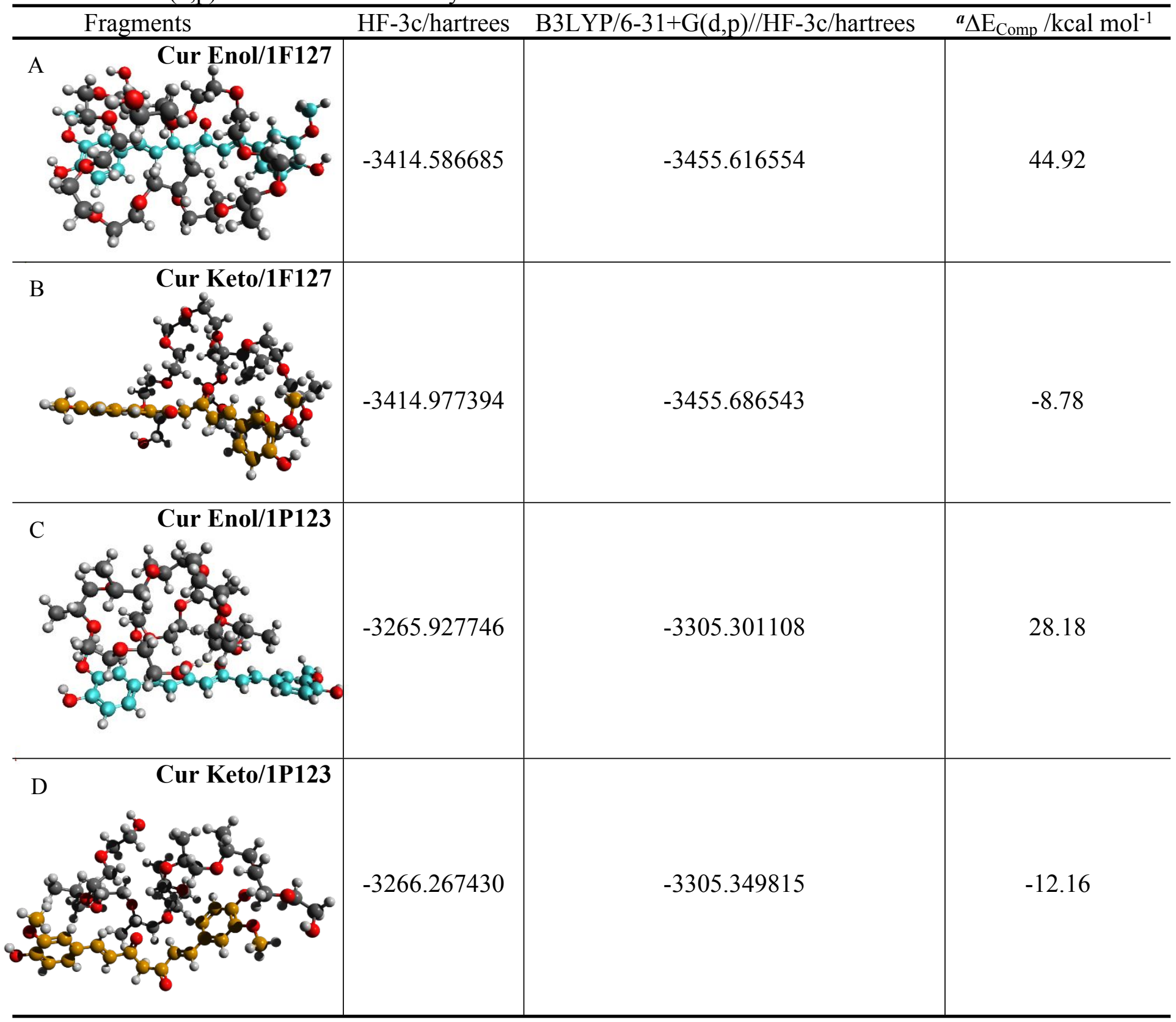

1 hartree $=627.5095 \mathrm{kcal} / \mathrm{mol}$.

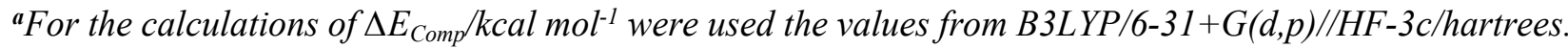


Table S5. Total electronic energy for the optimized structures of copolymeric fragments and copolymeric segments with Cur tautomers calculated at the HF-3c and B3LYP/6$31+\mathrm{G}(\mathrm{d}, \mathrm{p}) / / \mathrm{HF}-3 \mathrm{c}$ levels of theory in vacuum medium

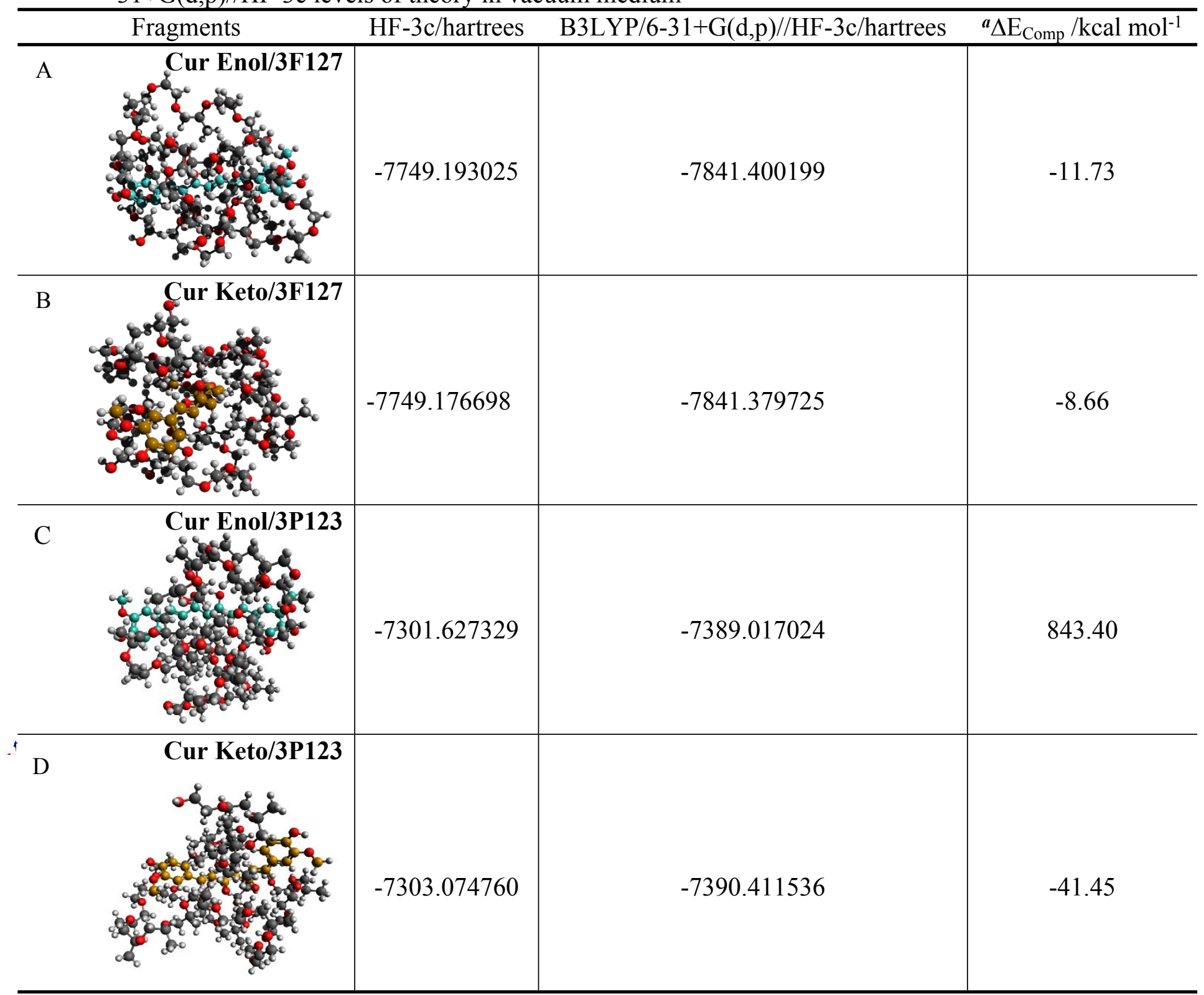

1 hartree $=627.5095 \mathrm{kcal} / \mathrm{mol}$.

${ }^{a} F$ or the calculations of $\Delta E_{\text {Comp }} / k$ al mol $^{-1}$ were used the values from $B 3 L Y P / 6-31+G(d, p) / / H F-3 c / h a r t r e e s$. 


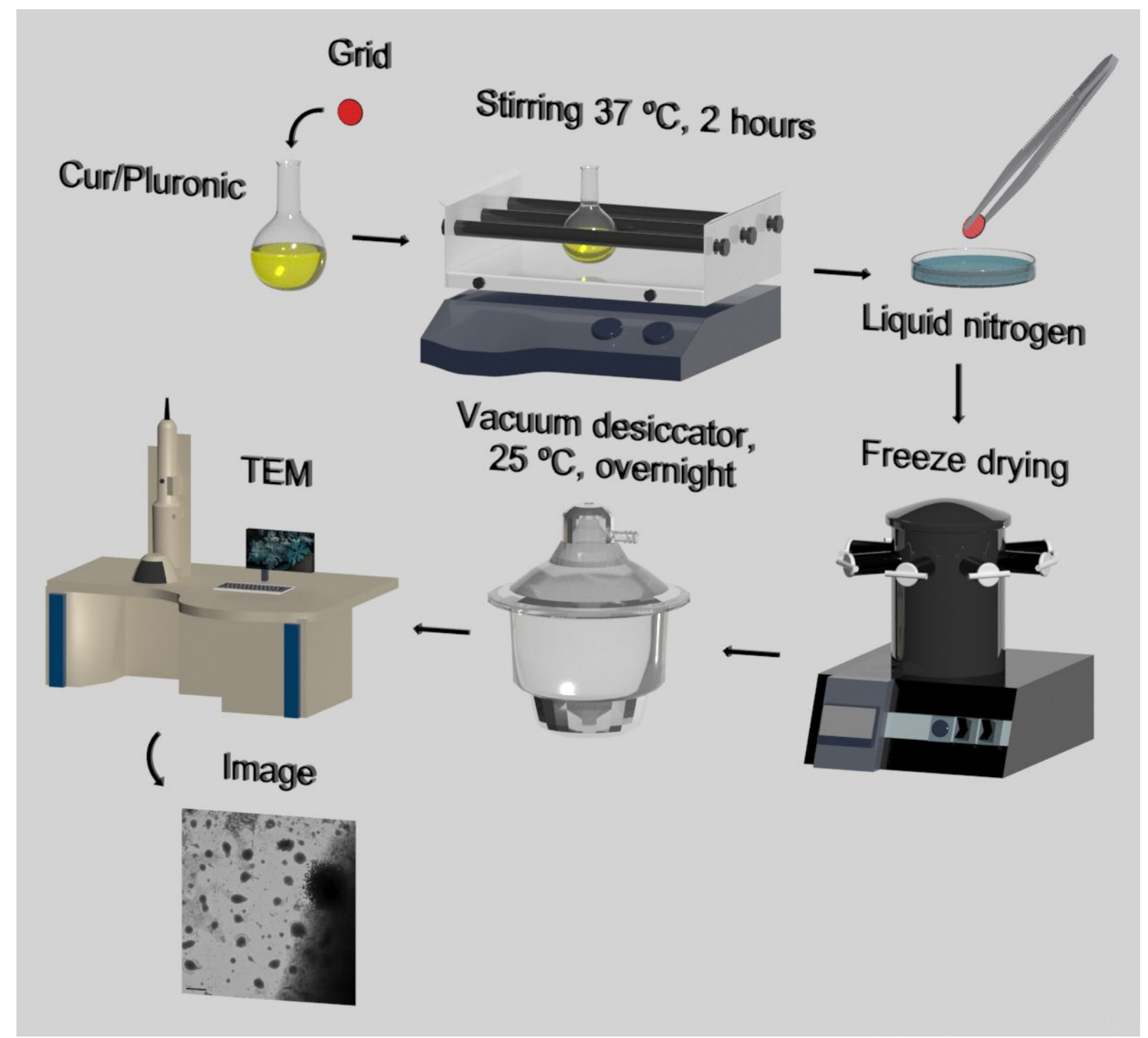

Figure S2. Preparation of the samples for the acquisition of micrographs by transmission electron microscopy.
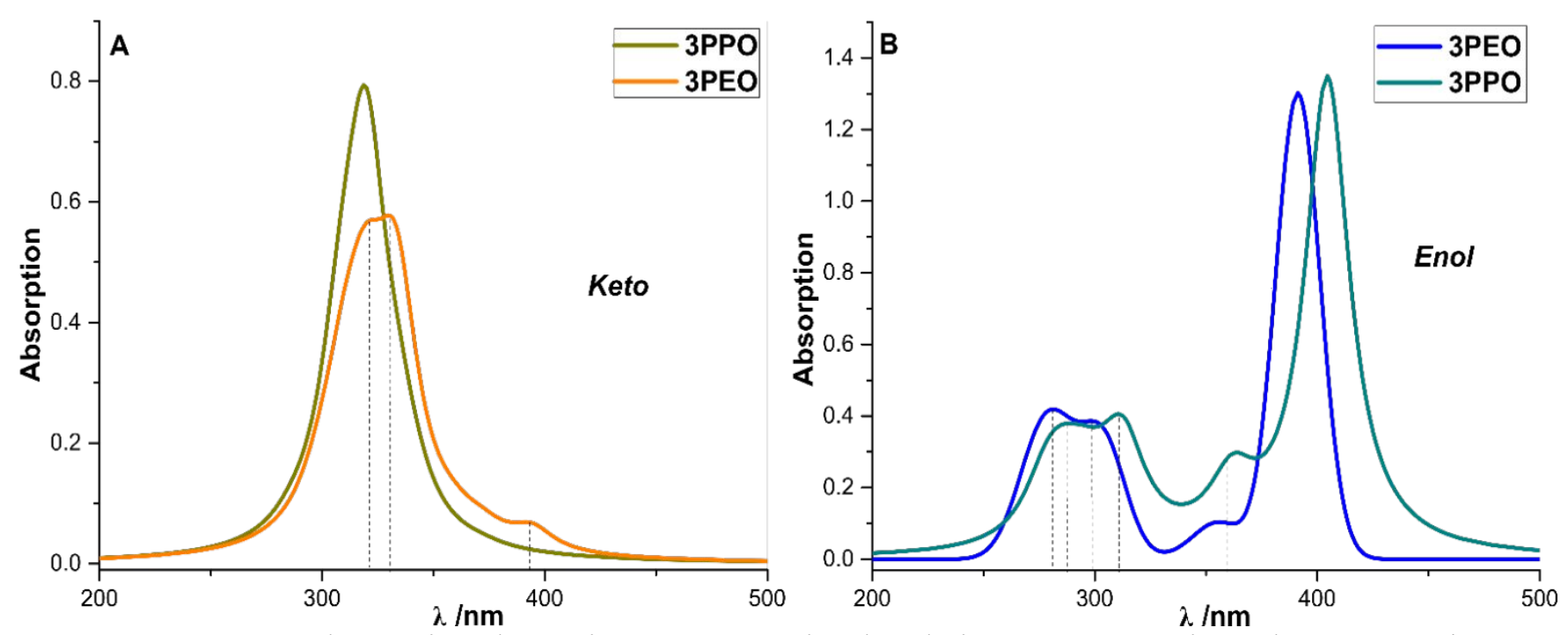

Figure S3. Electronic absorption spectra simulated in vacuum using the B3LYP/6$31+\mathrm{G}(\mathrm{d}, \mathrm{p}) / / \mathrm{HF}-3 \mathrm{c}$ levels of theory, being: A) Keto tautomeric form with 3 PEO and PPO fragments. B) Enol Tautomeric form with 3 PEO and PPO fragments. 
Table S6. Wavelengths values for the maximum absorption, oscillator strength $(f)$ and dipole moment $(\vec{\mu})$ for the complexes formed between the tautomers and the copolymeric blocks

\begin{tabular}{l|c|c|c|c|c|c|c|c|c}
\hline & $\lambda_{\mathbf{1}} / \mathrm{nm}$ & $\boldsymbol{f}$ & $\boldsymbol{\lambda}_{2} / \mathrm{nm}$ & $\boldsymbol{f}$ & $\boldsymbol{\lambda}_{3} / \mathrm{nm}$ & $\boldsymbol{f}$ & $\boldsymbol{\lambda}_{4} / \mathrm{nm}$ & $\boldsymbol{f}$ & $\overrightarrow{\boldsymbol{\mu}} /$ Debye \\
\hline Enol & 274 & 0.1534 & 298.6 & 0.06546 & 285 & 0.054611 & ------ & ------ & ------- \\
\hline Keto & 344 & 0.5390 & 346 & 0.002900 & 386 & 0.00008927 & 362 & 0.0000 & ------ \\
\hline Enol-3PEO & 391 & 1.3015 & 304 & 0.2990 & 284 & 0.2032 & 276 & 0.1820 & 2.2727 \\
\hline Enol-3PPO & 404 & 1.3274 & 313 & 0.2673 & 285 & 0.1342 & 277 & 0.1277 & $\mathbf{2 . 9 3 6 6}$ \\
\hline Keto-3PEO & 333 & 0.3932 & 320 & 0.2372 & 310 & 0.1726 & 394 & 0.03530 & 4.4302 \\
\hline Keto-3PPO & 320 & 0.5892 & 310 & 0.1768 & 333 & 0.1131 & ------ & ------ & $\mathbf{4 . 6 9 3 2}$ \\
\hline
\end{tabular}
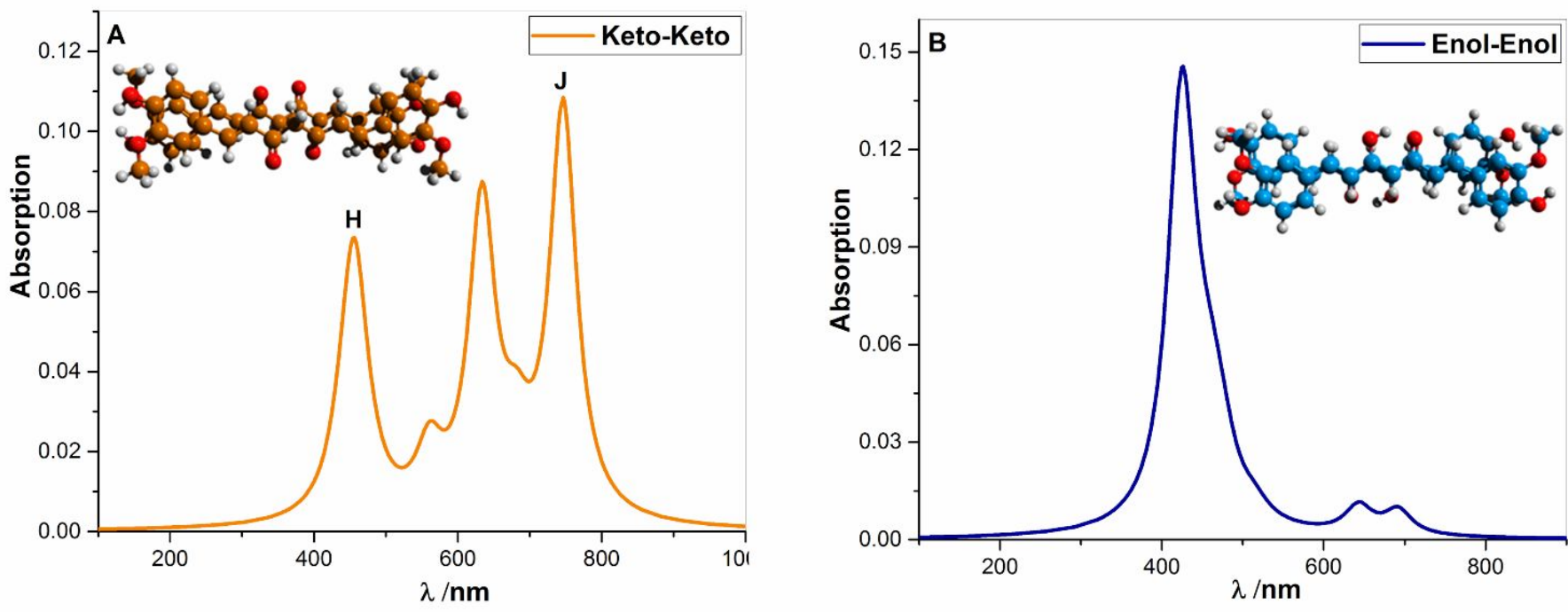

Figure S4. Theoretical electronic absorption spectra simulated in vacuum using the B3LYP/6$31+\mathrm{G}(\mathrm{d}, \mathrm{p}) / / \mathrm{HF}-3 \mathrm{c}$ levels of theory for the dimeric forms. A) Keto-Keto dimer and B) EnolEnol dimer. 
Table S7. Electronic transitions, wavelengths of absorption $(\lambda)$ and oscillator strength $(f)$ of the tautomers encapsulated in F127 and P123 triblock copolymers

\begin{tabular}{l|c|c|c|c|c|c|c|c|c}
\hline 3F127/Cur & $\lambda_{\mathbf{1}} / \mathrm{nm}$ & $\boldsymbol{f}$ & Transition & $\boldsymbol{\lambda}_{\mathbf{2}} / \mathrm{nm}$ & $\boldsymbol{f}$ & Transition & $\boldsymbol{\lambda}_{\mathbf{3}} / \mathrm{nm}$ & $\boldsymbol{f}$ & Transition \\
\hline Enol & 400 & 1.2647 & $\begin{array}{l}\mathrm{H} \rightarrow \mathrm{L} \\
\left(\boldsymbol{\pi} \rightarrow \boldsymbol{\pi}^{*}\right)\end{array}$ & 363 & 0.0466 & $\begin{array}{l}\mathrm{H}-1 \rightarrow \mathrm{L} \\
\left(\boldsymbol{\pi} \rightarrow \boldsymbol{\pi}^{*}\right)\end{array}$ & 341 & 0.0009 & $\begin{array}{l}\mathrm{H}-5 \rightarrow \mathrm{L} \\
\left(\mathbf{n} \rightarrow \boldsymbol{\pi}^{*}\right)\end{array}$ \\
\hline Keto & 357 & 0.0148 & $\begin{array}{l}\mathrm{H} \rightarrow \mathrm{L} \\
\left(\boldsymbol{\pi} \rightarrow \boldsymbol{\pi}^{*}\right)\end{array}$ & 349 & 0.2301 & $\begin{array}{l}\mathrm{H}-2 \rightarrow \mathrm{L}+1 \\
\left(\mathbf{n} \rightarrow \boldsymbol{\pi}^{*}\right)\end{array}$ & 339 & 0.0097 & $\begin{array}{l}\mathrm{H}-5 \rightarrow \mathrm{L} \\
\left(\mathbf{n} \rightarrow \boldsymbol{\pi}^{*}\right)\end{array}$ \\
\hline 3P123/Cur & $\boldsymbol{\lambda}_{\mathbf{1}} / \mathrm{nm}$ & $\boldsymbol{f}$ & Transition & $\boldsymbol{\lambda}_{\mathbf{2}} / \mathrm{nm}$ & $\boldsymbol{f}$ & Transition & $\boldsymbol{\lambda}_{\mathbf{3}} / \mathrm{nm}$ & $\boldsymbol{f}$ & Transition \\
\hline Enol & 400 & 1.1630 & $\begin{array}{l}\mathrm{H} \rightarrow \mathrm{L} \\
\left(\boldsymbol{\pi} \rightarrow \boldsymbol{\pi}^{*}\right)\end{array}$ & 365 & 0.0655 & $\begin{array}{l}\mathrm{H}-1 \rightarrow \mathrm{L} \\
\left(\boldsymbol{\pi} \rightarrow \boldsymbol{\pi}^{*}\right)\end{array}$ & 344 & 0.0032 & $\begin{array}{c}\mathrm{H}-5 \rightarrow \mathrm{L} \\
\left(\mathbf{n} \rightarrow \boldsymbol{\pi}^{*}\right)\end{array}$ \\
\hline Keto & 359 & 0.0312 & $\begin{array}{c}\mathrm{H} \rightarrow \mathrm{L} \\
\left(\boldsymbol{\pi} \rightarrow \boldsymbol{\pi}^{*}\right)\end{array}$ & 347 & 0.0242 & $\begin{array}{l}\mathrm{H}-2 \rightarrow \mathrm{L} \\
\left(\mathbf{n} \rightarrow \boldsymbol{\pi}^{*}\right)\end{array}$ & 340 & 0.0734 & $\begin{array}{c}\mathrm{H}-2 \rightarrow \mathrm{L}+1 \\
\left(\mathbf{n} \rightarrow \boldsymbol{\pi}^{*}\right)\end{array}$ \\
\hline
\end{tabular}

$H=$ Homo orbital, $L=$ Lumo orbital, *anti ligand orbital, $f=$ oscillator strength. 

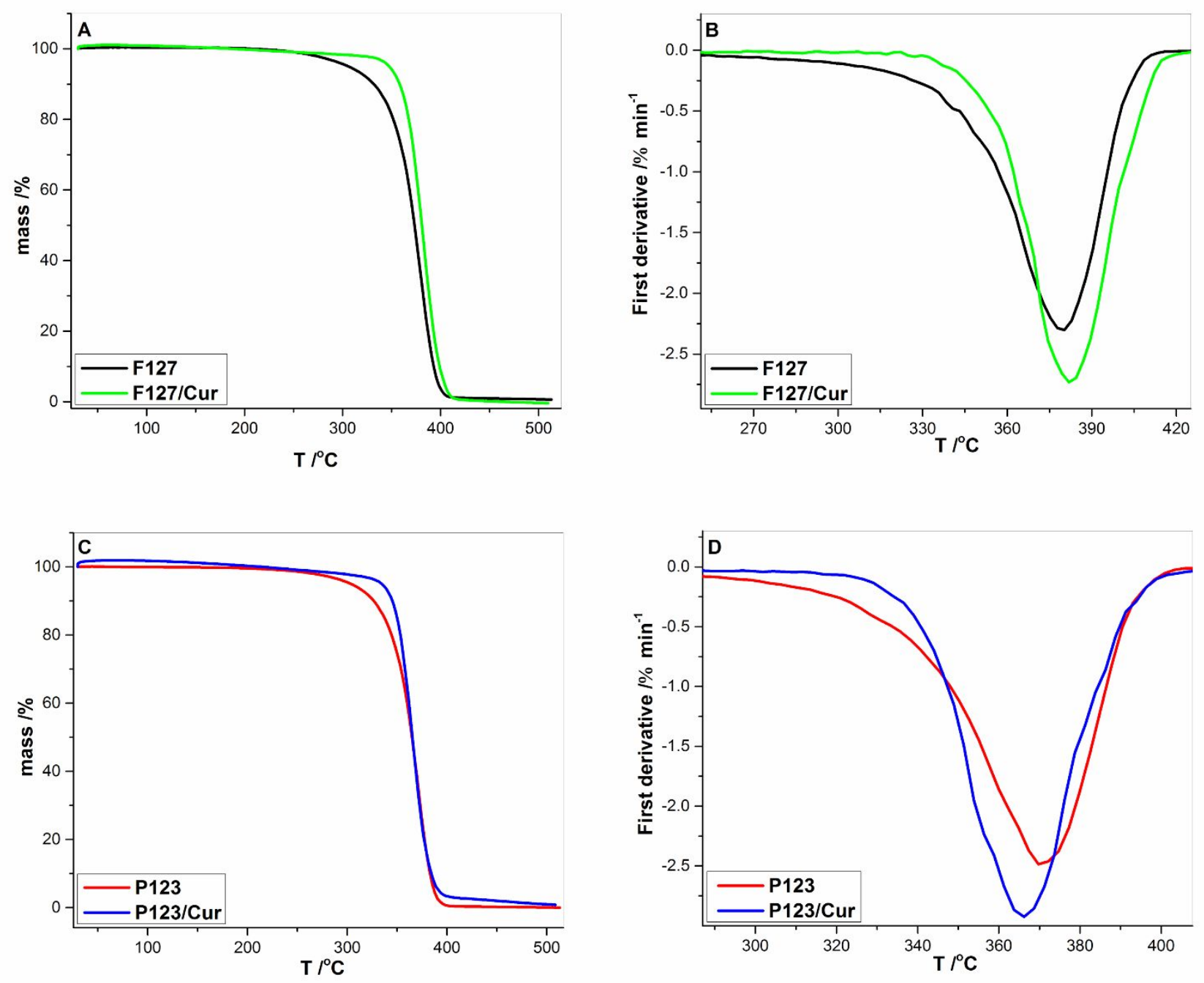

Figure S5. Thermogram and first derivative of copolymers P123, F127 and formulated P123/Cur and F127/Cur.

Table S8. $\mathrm{T}_{\text {Deg }}$ of P123, F127 copolymers and formulated P123/Cur and F127/Cur

\begin{tabular}{l|c}
\hline Materials & $\mathbf{T}_{\text {Deg }} /{ }^{\circ} \mathrm{C}$ \\
\hline F127 & 379.8 \\
\hline F127/Cur & 382.8 \\
\hline P123 & 370.9 \\
\hline P123/Cur & 366.0 \\
\hline
\end{tabular}



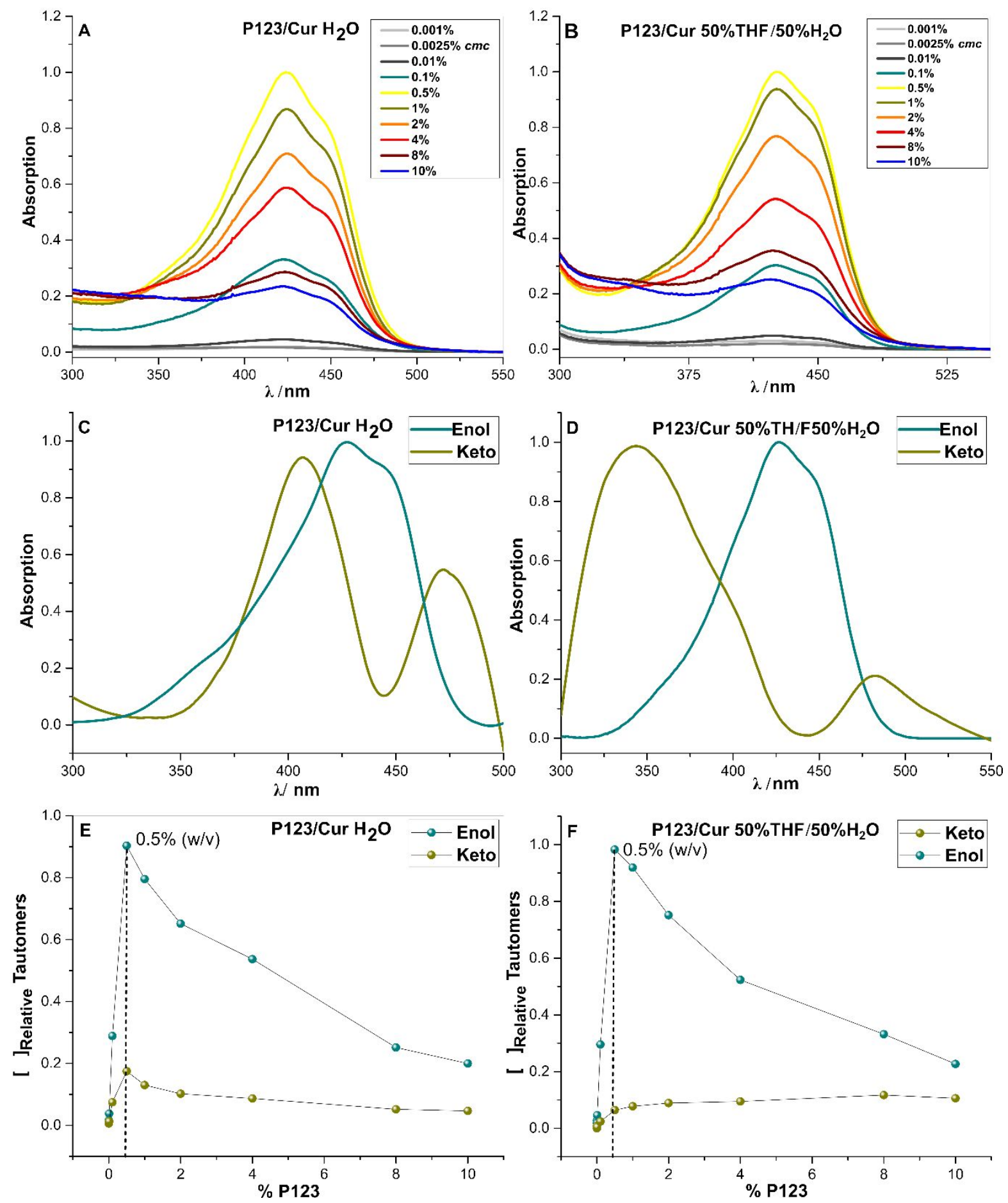

Figure S6. A-B) Electronic absorption spectra for the formulated P123/Cur in water and in the mixture of $50 \% \mathrm{THF} / 50 \% \mathrm{H}_{2} \mathrm{O}$ as a function of increased P123 concentration. C-D) Experimental recovered electronic absorption spectra of Keto and Enol tautomers for the formulated P123/Cur in water and 50\% THF/50\% $\mathrm{H}_{2} \mathrm{O}$. E-F) Relative concentration of tautomeric species in water and $50 \% \mathrm{THF} / 50 \% \mathrm{H}_{2} \mathrm{O}$ as a function of the $\mathrm{P} 123$ ratio. 

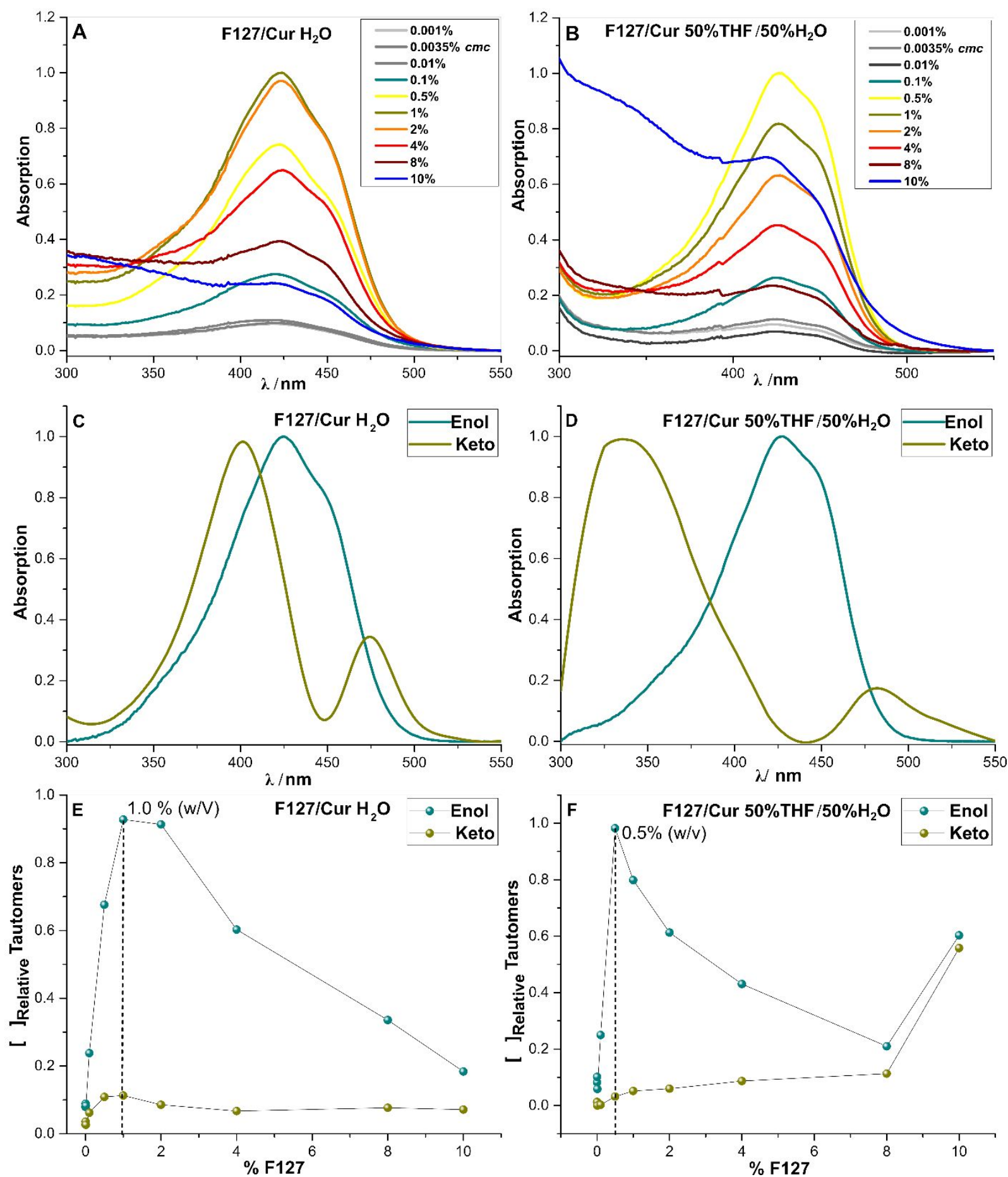

Figure S7. A-B) Electronic absorption spectra for the formulated F127/Cur in water and in the mixture of $50 \% \mathrm{THF} / 50 \% \mathrm{H}_{2} \mathrm{O}$ as a function of increased $\mathrm{F} 127$ concentration. C-D) Experimental recovered electronic absorption spectra of Keto and Enol tautomers for the formulated F127/Cur in water and in 50\% THF/50\% $\mathrm{H}_{2} \mathrm{O}$. E-F) Relative concentration of tautomeric species in water and $50 \% \mathrm{THF} / 50 \% \mathrm{H}_{2} \mathrm{O}$ as a function of the $\mathrm{F} 127$ ratio. 
Table S9. Electronic transitions, wavelengths of absorption $(\lambda)$ and oscillator strength $(f)$ of Keto-Keto and Enol-Enol tautomeric dimers

\begin{tabular}{|c|c|c|c|c|c|c|c|c|c|}
\hline Dimer & $\lambda_{1} / \mathrm{nm}$ & $\bar{f}$ & Transition & $\lambda_{2} / \mathrm{nm}$ & $\bar{f}$ & Transition & $\lambda_{3} / \mathrm{nm}$ & $\bar{f}$ & Transition \\
\hline Enol-Enol & 432 & 0.0018 & $\begin{array}{l}\mathrm{H} \rightarrow \mathrm{L}+1 \\
\left(\boldsymbol{\pi} \rightarrow \boldsymbol{\pi}^{*}\right) \\
\mathrm{H}-1 \rightarrow \mathrm{L}+1 \\
\left(\boldsymbol{\pi} \rightarrow \boldsymbol{\pi}^{*}\right)\end{array}$ & 425 & 0.0198 & $\begin{array}{l}\mathrm{H} \rightarrow \mathrm{L}+1 \\
\left(\boldsymbol{\pi} \rightarrow \boldsymbol{\pi}^{*}\right) \\
\mathrm{H} \rightarrow \mathrm{L} \\
\left(\boldsymbol{\pi} \rightarrow \boldsymbol{\pi}^{*}\right)\end{array}$ & 423 & 0.0406 & $\begin{array}{l}\mathrm{H} \rightarrow \mathrm{L}+1 \\
\left(\boldsymbol{\pi} \rightarrow \pi^{*}\right) \\
\mathrm{H}-1 \rightarrow \mathrm{L} \\
\left(\boldsymbol{\pi} \rightarrow \pi^{*}\right)\end{array}$ \\
\hline Keto-Keto & 378 & 0.0001 & $\begin{array}{l}\mathrm{H}-1 \rightarrow \mathrm{L}+1 \\
\left(\boldsymbol{\pi} \rightarrow \boldsymbol{\pi}^{*}\right) \\
\mathrm{H}-2 \rightarrow \mathrm{L}+1 \\
\left(\boldsymbol{\pi} \rightarrow \boldsymbol{\pi}^{*}\right) \\
\mathrm{H}-5 \rightarrow \mathrm{L} \\
\left(\mathbf{n} \rightarrow \boldsymbol{\pi}^{*}\right)\end{array}$ & 372.90 & 0.0091 & $\begin{array}{l}\mathrm{H}-1 \rightarrow \mathrm{L}+2 \\
\left(\boldsymbol{\pi} \rightarrow \boldsymbol{\pi}^{*}\right) \\
\mathrm{H}-1 \rightarrow \mathrm{L} \\
\left(\boldsymbol{\pi} \rightarrow \boldsymbol{\pi}^{*}\right)\end{array}$ & 372.40 & 0.0339 & $\begin{array}{l}\mathrm{H} \rightarrow \mathrm{L}+1 \\
\left(\boldsymbol{\pi} \rightarrow \boldsymbol{\pi}^{*}\right) \\
\mathrm{H} \rightarrow \mathrm{L} \\
\left(\boldsymbol{\pi} \rightarrow \boldsymbol{\pi}^{*}\right) \\
\mathrm{H}-1 \rightarrow \mathrm{L}+3 \\
\left(\boldsymbol{\pi} \rightarrow \boldsymbol{\pi}^{*}\right) \\
\mathrm{H}-3 \rightarrow \mathrm{L}+1 \\
\left(\boldsymbol{\pi} \rightarrow \boldsymbol{\pi}^{*}\right)\end{array}$ \\
\hline
\end{tabular}

$H=$ Homo orbital, $L=$ Lumo orbital, *anti ligand orbital, $f=$ oscillator strength. 
Table S10. Molecular orbitals referring to the electronic transitions of the Keto-Keto and EnolEnol tautomeric dimers

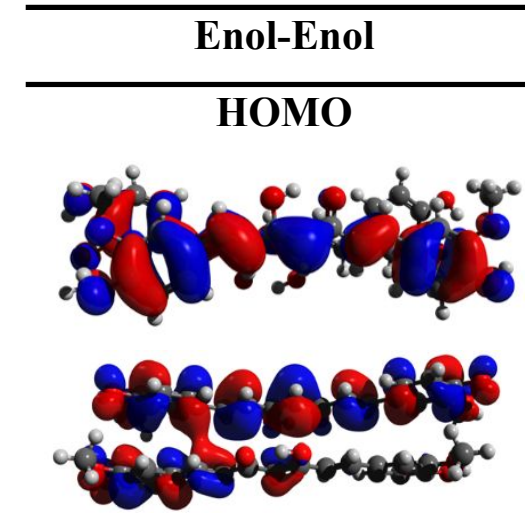

HOMO-1

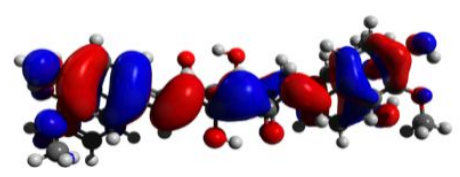

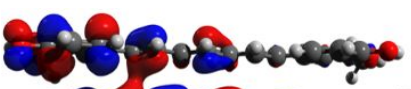
-

Keto-Keto

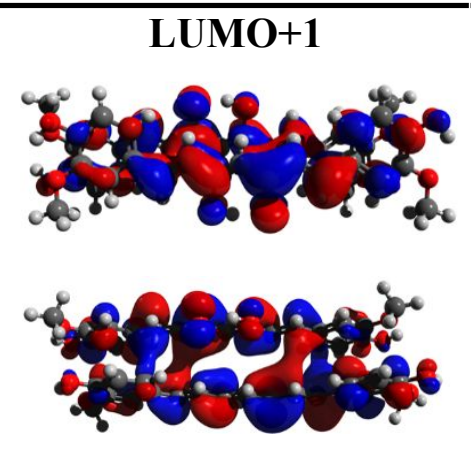

LUMO

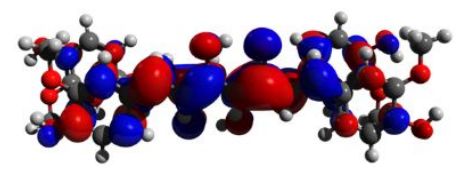
asent inase

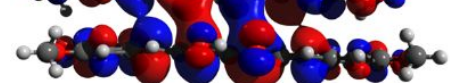

HOMO
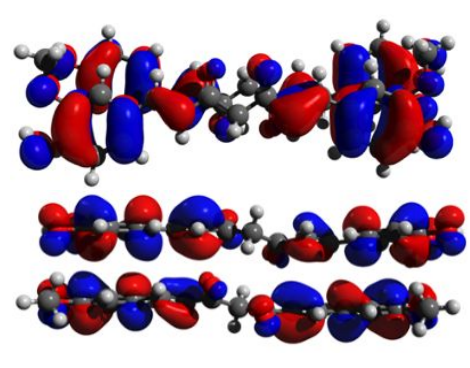

HOMO-1

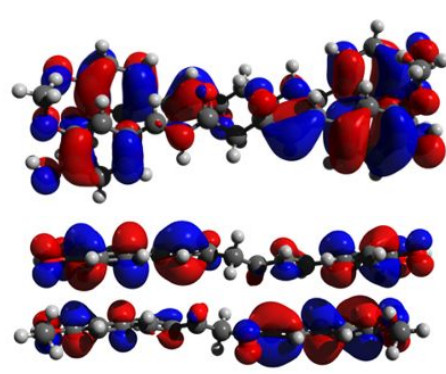

HOMO-2

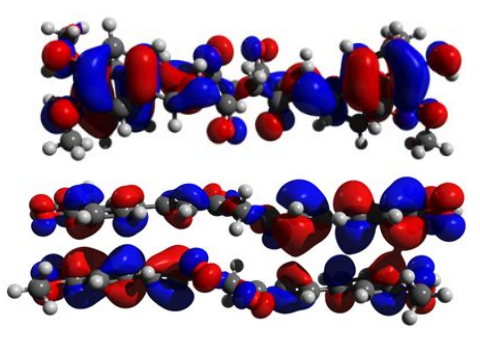

HOMO-3

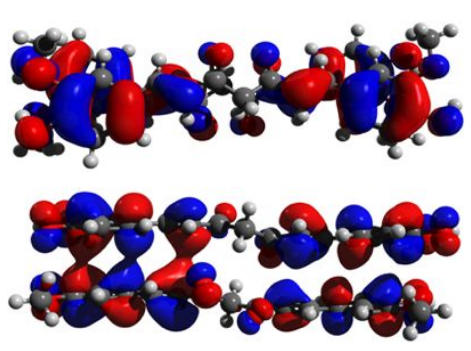

HOMO-5
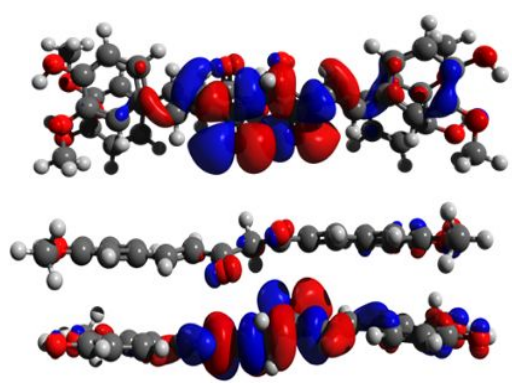

\section{LUMO+3}

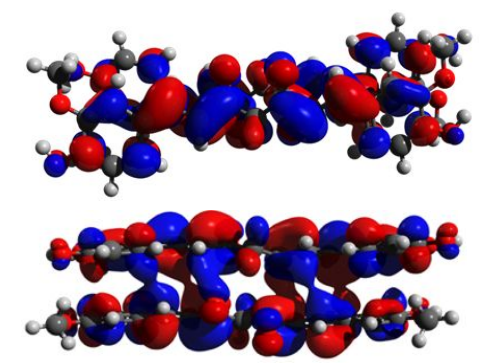

LUMO+2

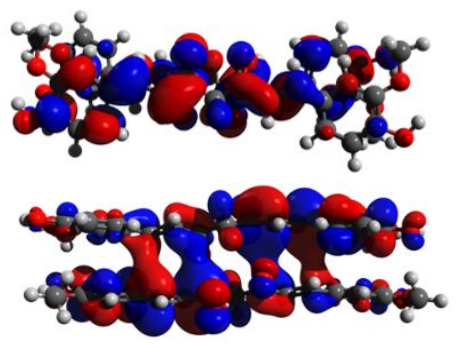

$$
\text { LUMO+1 }
$$

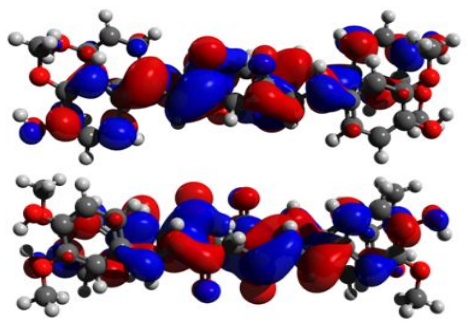

LUMO

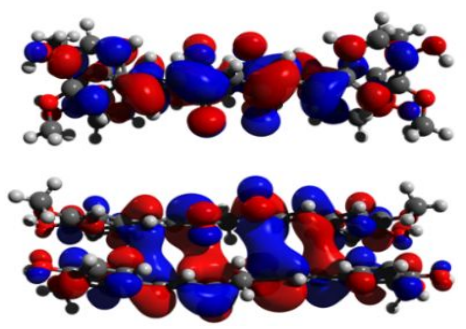




\section{A) Keto-Keto}
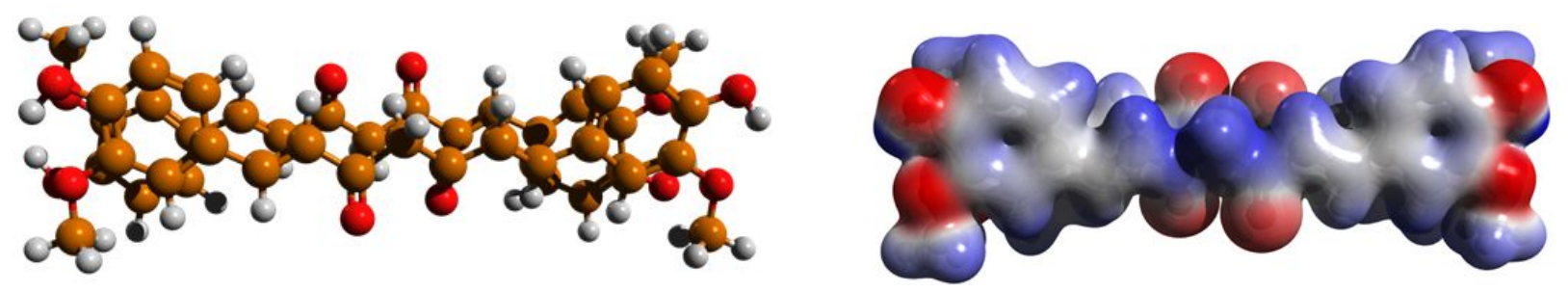

B) Enol-Enol
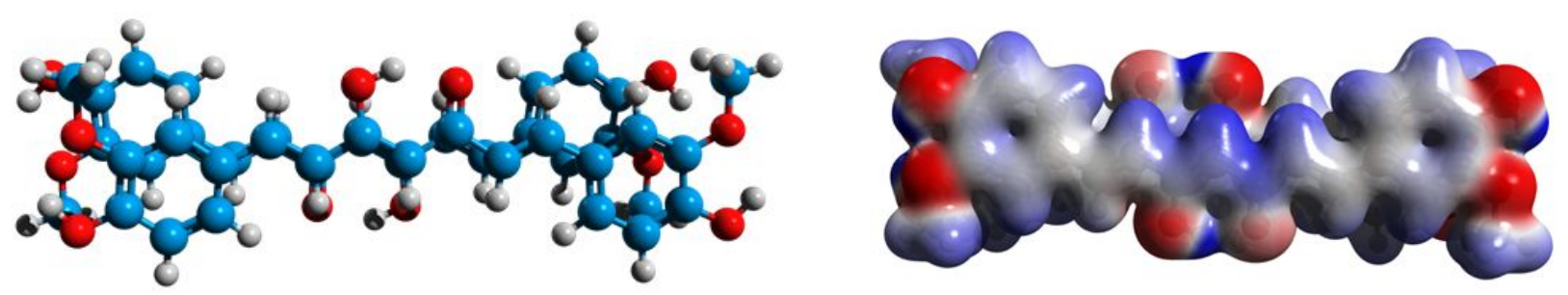

C)

Enol-Enol

Keto-Keto
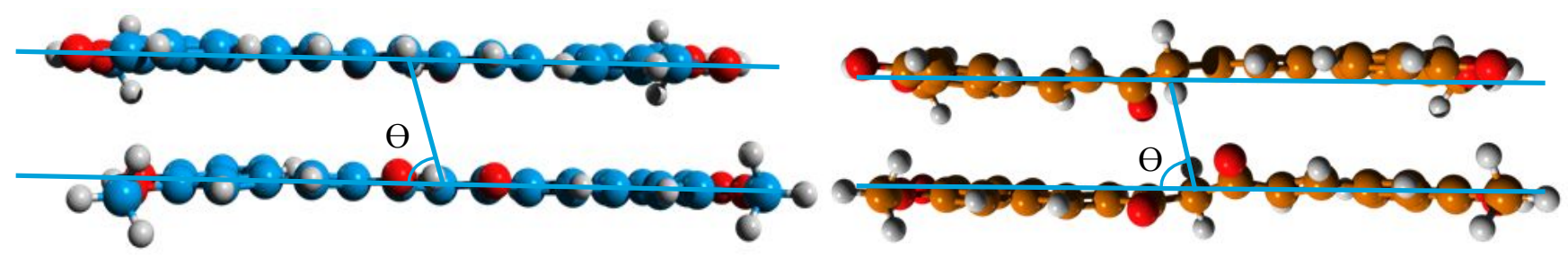<smiles>COc1cc(/C=C/C2COCOC2/C=C/c2ccc(O)c(OC)c2)ccc1O</smiles>

Figure S8. A) Optimized structure of the Cur dimer for the Keto tautomer and its electrostatic potential map. B) Optimized structure of the Cur dimer for the Enol tautomer and its electrostatic potential map. C) Illustrative angle formed between the Keto-Keto and Enol-Enol dimers. Images were generated with molecular mechanics force field by the Avogadro 1.1.1 program, with $0.015 \mathrm{e} / \AA^{3}$ isovalue for the map of electrostatic potential. The reddish region is the most negative while the bluish region is the most positive. 


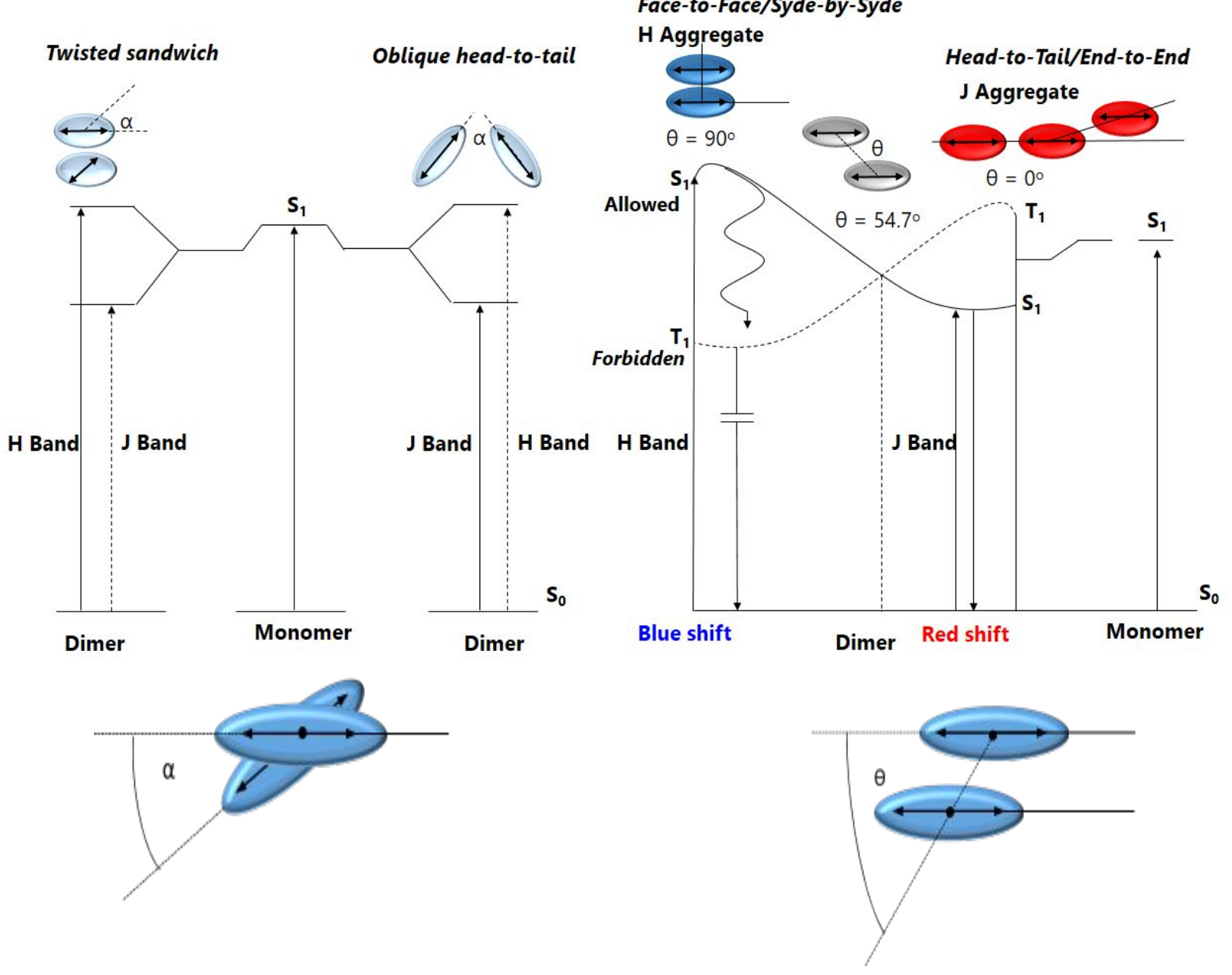

Figure S9. Energy diagram for dimers based on the Exciton theory ${ }^{1,2,3,4,5,6,7}$.

(1) Kasha, M. Energy Transfer Mechanisms and the Molecular Exciton Model for Molecular Aggregates. Radiat. Res. 1963, 20 (1), 55. https://doi.org/10.2307/3571331.

(2) Kasha, M.; Rawls, H. R.; Ashraf El-Bayoumi, M. The Exciton Model in Molecular Spectroscopy. Pure Appl. Chem. 1965, 11 (3-4), 371-392. https://doi.org/10.1351/pac196511030371.

(3) Seki, T.; Ichimura, K. Formation of Head-to-Tail and Side-by-Side Aggregates of Photochromic Spiropyrans in Bilayer Membrane. J. Phys. Chem. 1990, 94 (9), 3769-3775. https://doi.org/10.1021/j100372a077.

(4) Würthner, F.; Kaiser, T. E.; Saha-Möller, C. R. J-Aggregates: From Serendipitous Discovery to Supramolecular Engineering of Functional Dye Materials. Angew. Chemie Int. Ed. 2011, 50 (15), 3376-3410. https://doi.org/10.1002/anie.201002307.

(5) Toncelli, C.; Pino-Pinto, J. P.; Sano, N.; Picchioni, F.; Broekhuis, A. A.; Nishide, H.; MorenoVilloslada, I. Controlling the Aggregation of 5,10,15,20-Tetrakis-(4-Sulfonatophenyl)Porphyrin by the Use of Polycations Derived from Polyketones Bearing Charged Aromatic Groups. Dye. Pigment. 2013, 98 (1), 51-63. https://doi.org/10.1016/j.dyepig.2013.01.008.

(6) Klymchenko, A. S. Emerging Field of Self-Assembled Fluorescent Organic Dye Nanoparticles Emerging Field of Self-Assembled Fluorescent Organic Dye Nanoparticles. 2013, 3 (21), 1-8. Retrieved from https://scholar.google.com/scholar_lookup?journal=J.+Nanosci. +Lett\&title=Emerging + field + of + selfassembled + fluorescent + organic + dye + nanoparticles\&author $=\mathrm{AS}+\mathrm{Klym}$ chenko\&volum $\mathrm{e}=3$ \&issue $=21$ \&publication year $=2013$ \&pages $=1-8 \&$

(7) Arık, M.; Kassa, S. B.; Onganer, Y. Molecular Aggregates of Pyronin Dyes with Polyelectrolyte Polystyrene Sulfonate (PSS) in Aqueous Solution. J. Photochem. Photobiol. A Chem. 2020, 391 
(December 2019), 112309. https://doi.org/10.1016/j.jphotochem.2019.112309.
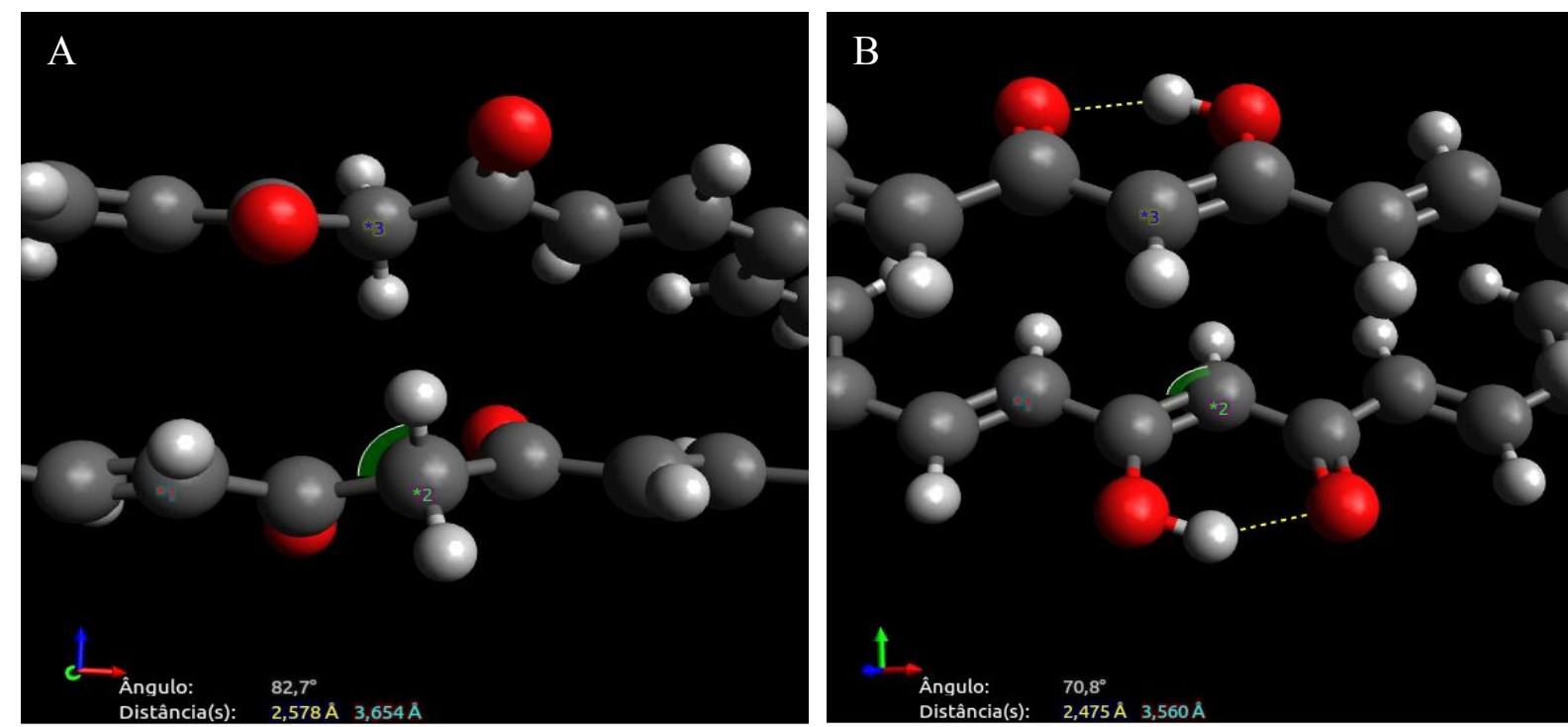

Figure S10. Angles and molecular distances between Keto-Keto and Enol-Enol dimers using advanced molecular editor Avogadro program, version 1.1.1. A) Keto-Keto dimer. B) EnolEnol dimer.
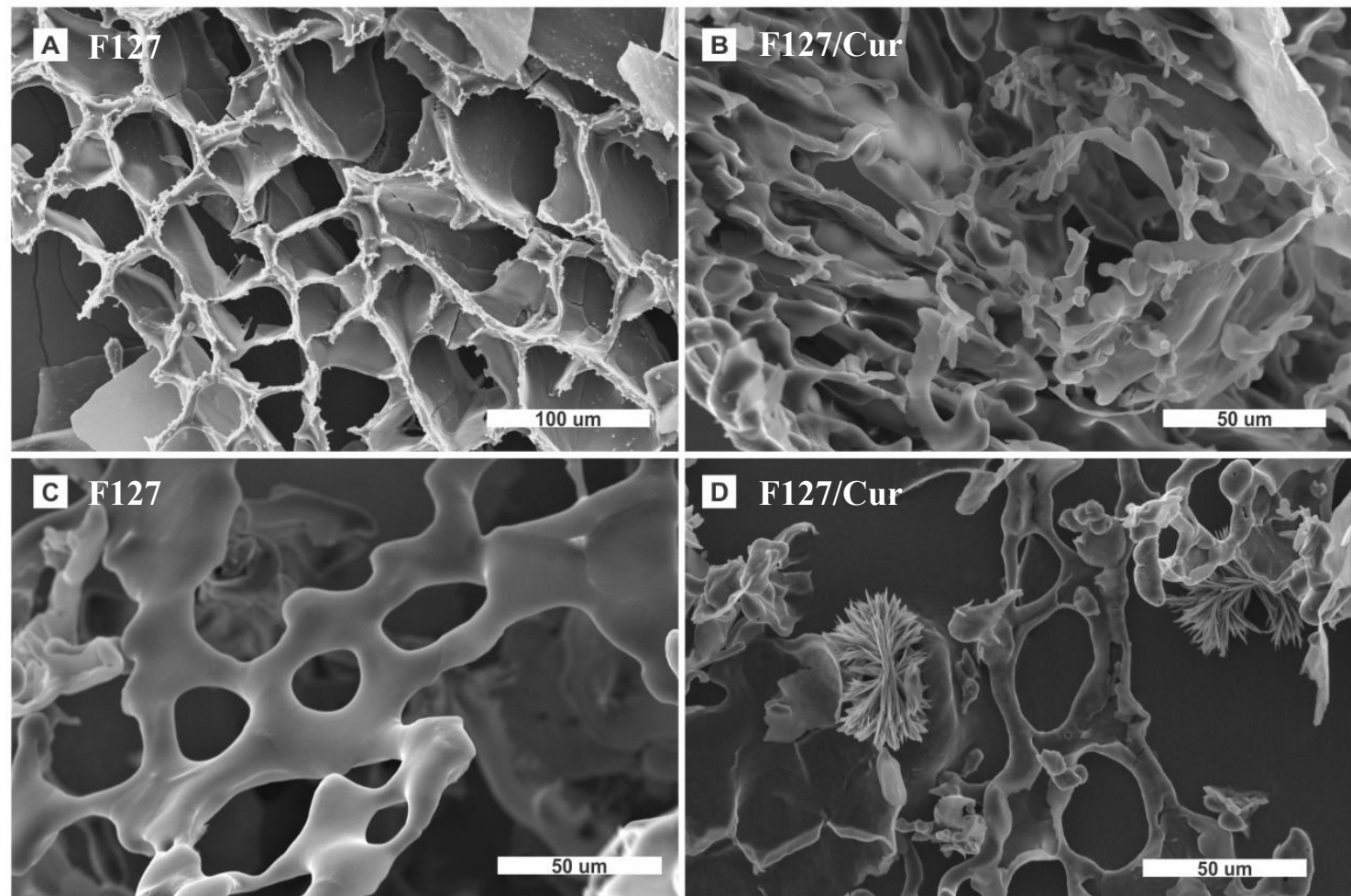

Figure S11. Comparison between scanning electron microscopies of copolymer F127 (A and C) and for the $500 \mu \mathrm{mol} \mathrm{L}-1$ of Cur formulated in F127/Cur (B and D). 


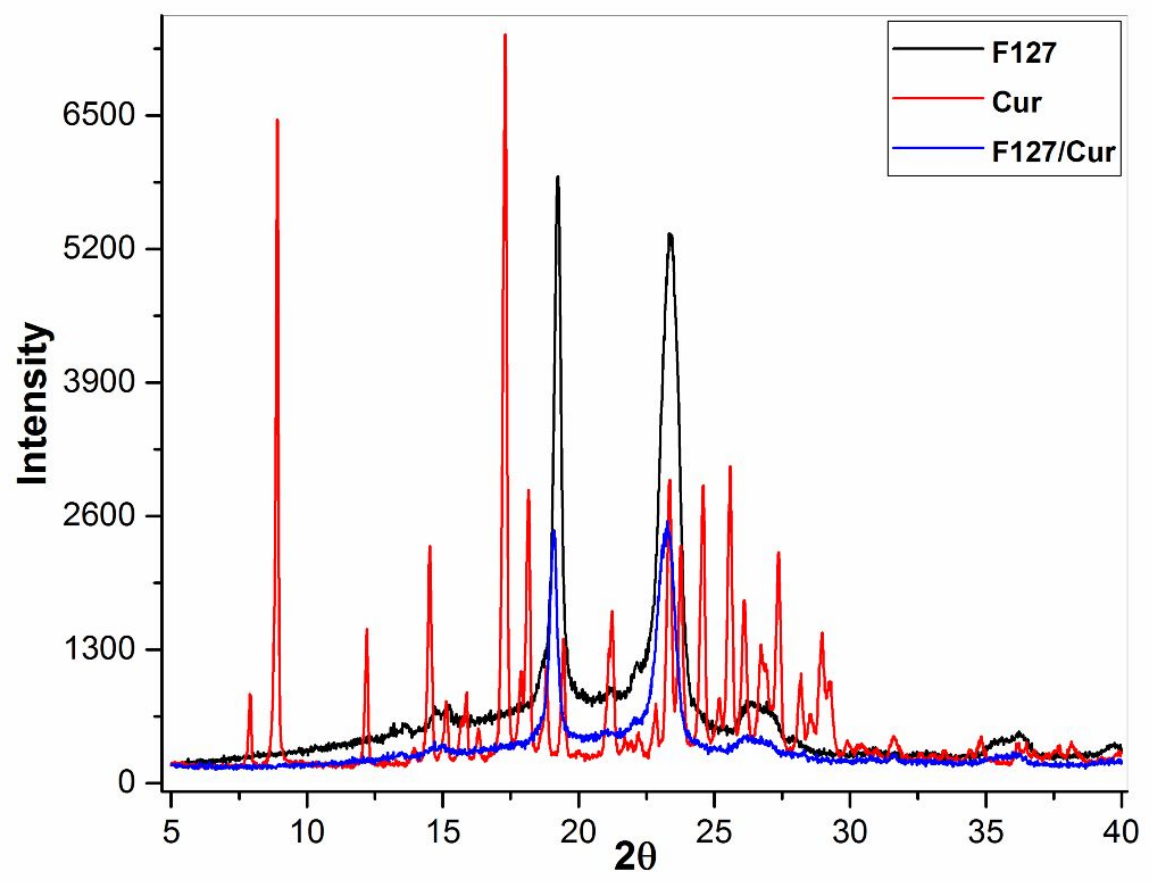

Figure S12. Cur diffractograms of F127 copolymer and formulated F127/Cur. 


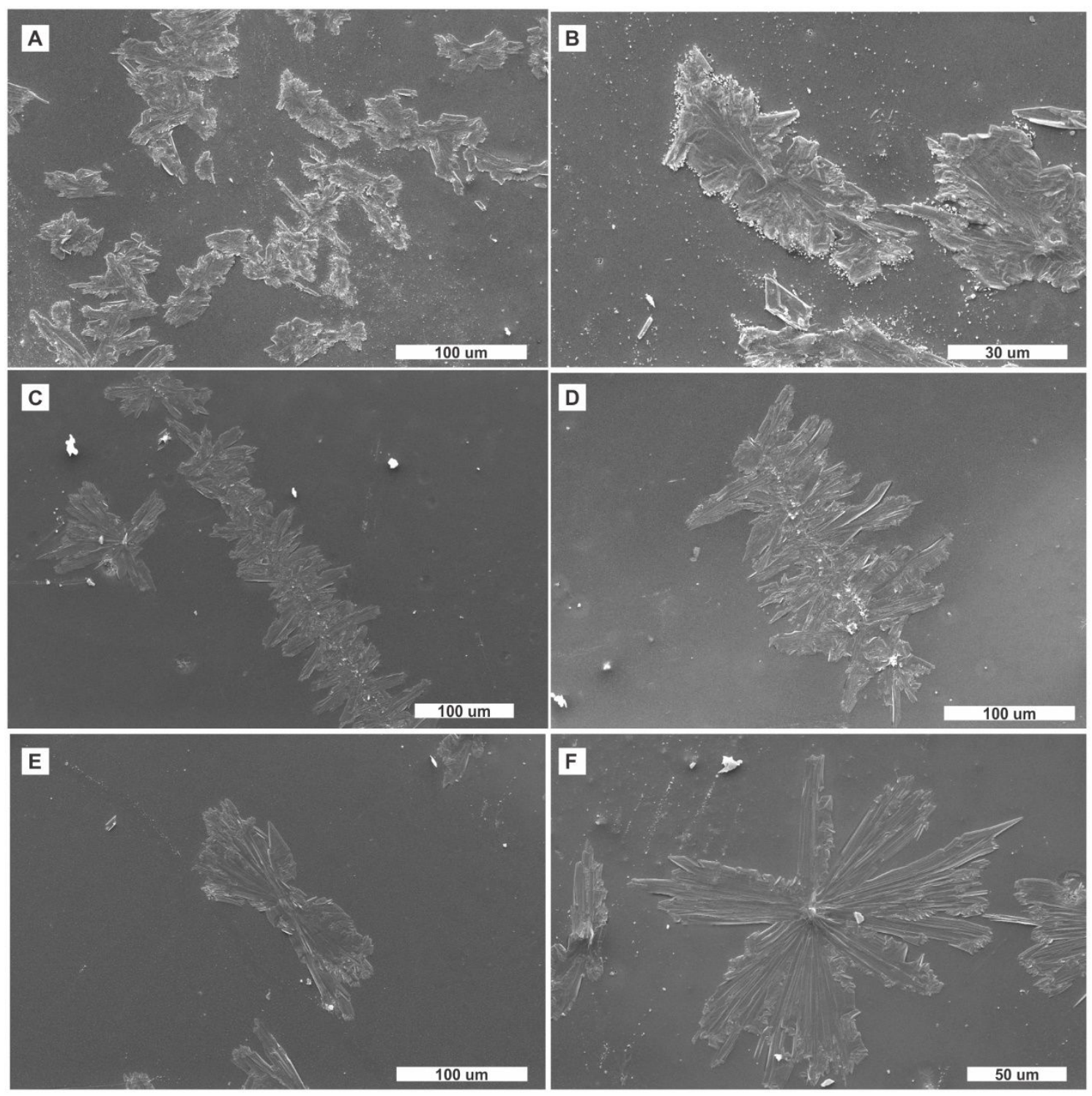

Figure S13. Scanning electron microscopies of crystallized Cur $\left(500.0 \mu \mathrm{mol} \mathrm{L}^{-1}\right)$ from ethanol evaporation $(\mathrm{A}-\mathrm{F})$. 


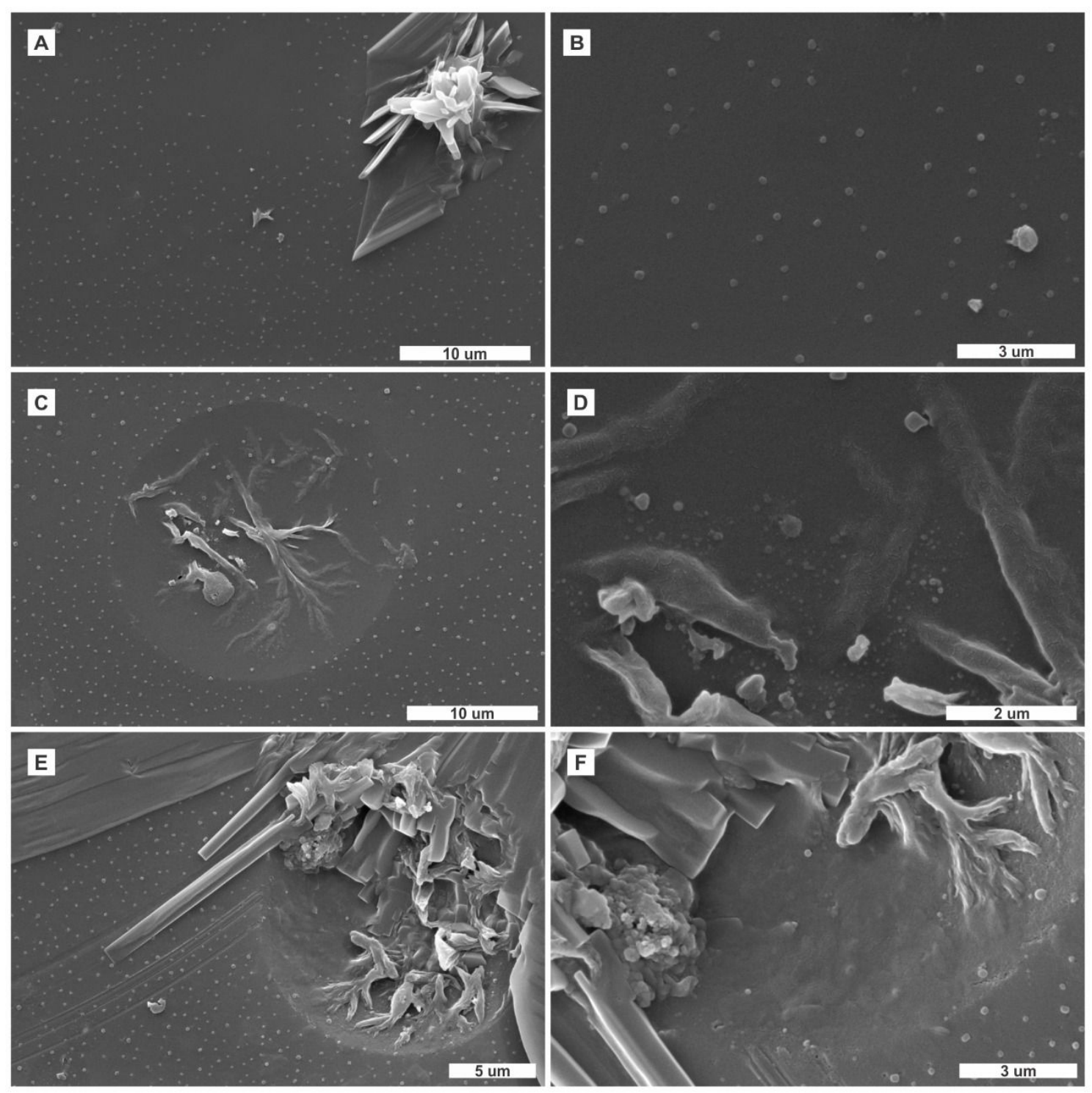

Figure S14. Scanning electron microscopies of crystallized Cur $\left(500.0 \mu \mathrm{mol} \mathrm{L}^{-1}\right)$ from ethanol evaporation (A-F). 

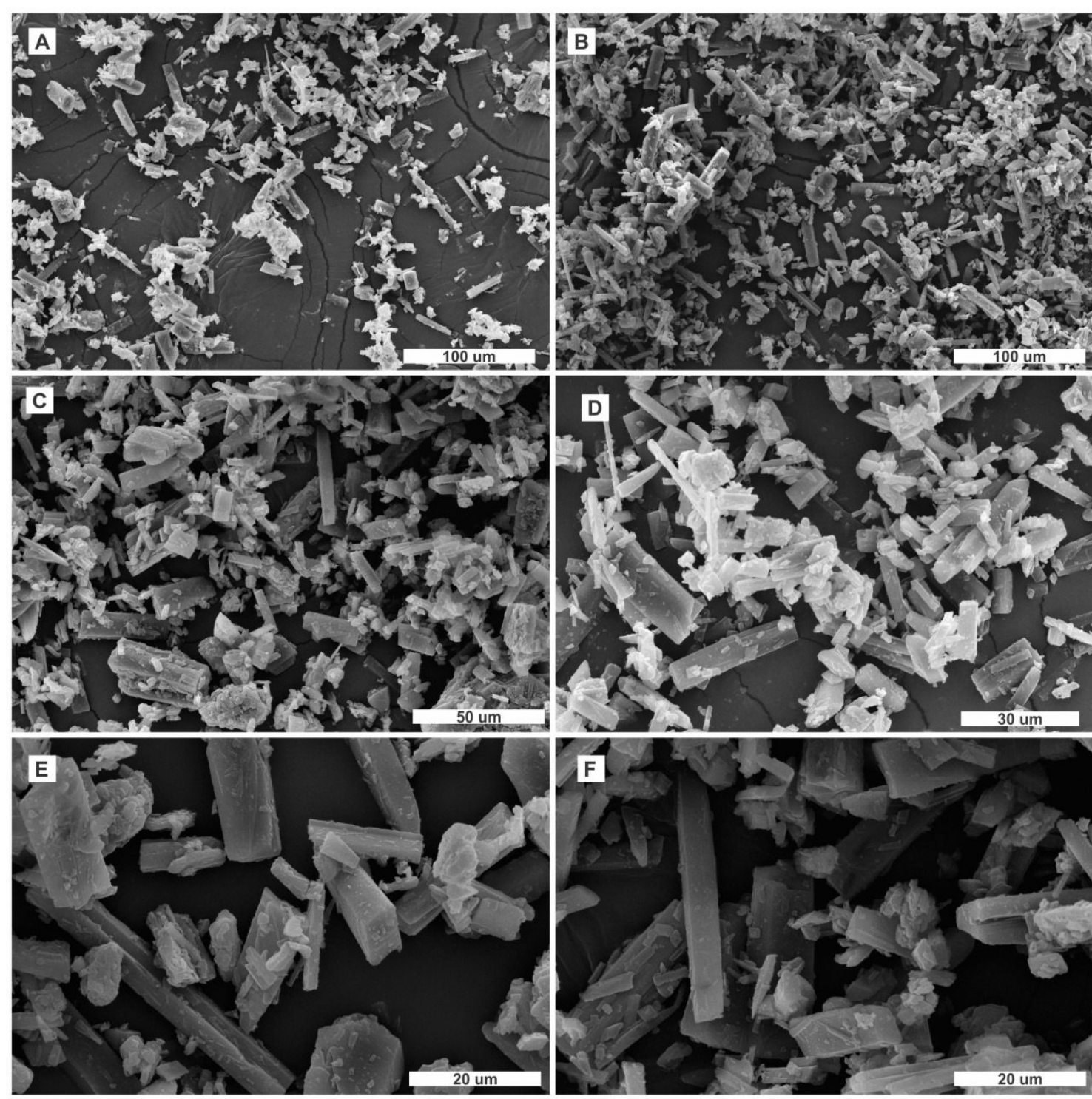

Figure S15. Scanning electron microscopies of Cur from stock (A-F). 

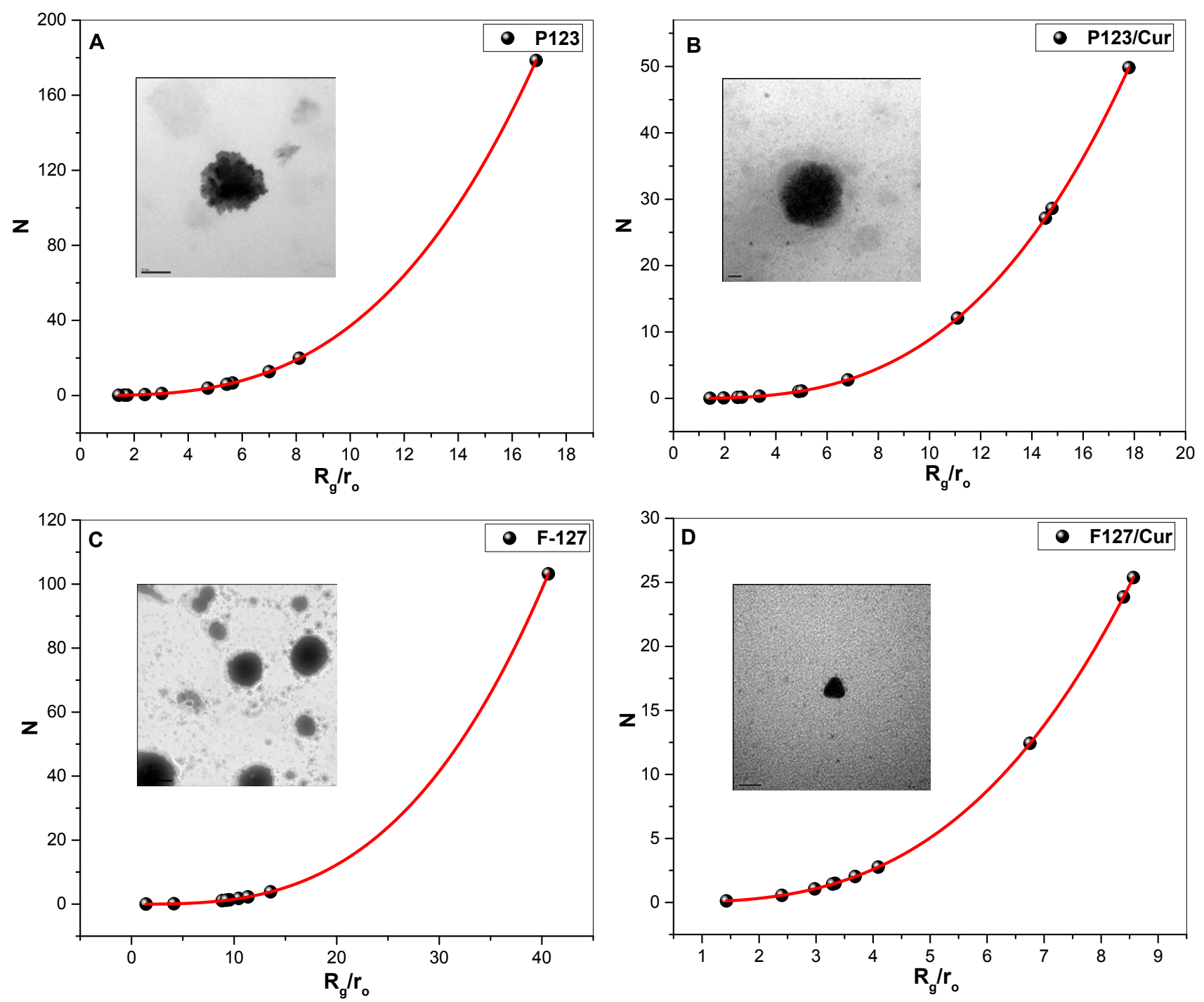

Figure S16. $\mathrm{N}$ for $\mathrm{R}_{\mathrm{g}} / \mathrm{r}_{\mathrm{o}}$ ratio to determine fractal dimension values of formulated copolymers P123 (A), F127 (C) and P123/Cur (B) and F127/Cur (D). 